\title{
Analysis of Metal Transfer and Weld Forming Characteristics in Triple-wire Gas Indirect Arc Welding
}

Liming Liu ( $\boldsymbol{D}$ liulm@dlut.edu.cn )

Key Laboratory of Liaoning Advanced Welding and Joining Technology

\section{Zeli Wang}

Key Laboratory of Liaoning Advanced Welding and Joining Technology

Tianyi Zhang

Key Laboratory of Liaoning Advanced Welding and Joining Technology

\section{Xianli Ba}

Key Laboratory of Liaoning Advanced Welding and Joining Technology

\section{Research Article}

Keywords: Triple-wire gas indirect arc welding, metal transfer, energy conversion, arc conductive channel

Posted Date: December 13th, 2021

DOI: https://doi.org/10.21203/rs.3.rs-1154158/v1

License: (a) (i) This work is licensed under a Creative Commons Attribution 4.0 International License. Read Full License

Version of Record: A version of this preprint was published at The International Journal of Advanced Manufacturing Technology on April 18th, 2022. See the published version at https://doi.org/10.1007/s00170-022-09119-x. 


\title{
Analysis of metal transfer and weld forming characteristics in triple-wire gas indirect arc welding
}

\author{
Liming Liu ${ }^{1} \cdot$ Zeli Wang $^{1} \cdot$ Tianyi Zhang ${ }^{1} \cdot$ Xianli Ba $^{1}$ \\ ${ }^{1}$ Liaoning Key Laboratory of Advanced Welding and Joining Technology, Dalian University of \\ Technology, Dalian, 116024, China \\ Corresponding author: Liming Liu

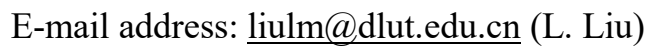

\section{Abstract}

Triple-wire gas indirect arc welding (TW-GIA) has the advantages of low heat input and high deposition rate. However, the simultaneous melting of triple wires makes the metal transfer mode complicated. The unknown of the metal transfer mode restricts the development of this technology. In this paper, high-speed camera systems and electrical signal acquisition sensors were used to explore the TW-GIA metal transfer mode. The static force model and the arc conductive channel model were used to discuss the droplet force and energy conversion characteristics respectively. Results showed that the TW-GIA metal transfer modes can be divided into: short-circuit transfer (SCT), main wire projected transfer + side wire globular transfer (PGT), main wire streaming transfer + side wire projected transfer $(\mathrm{SPT})$ and main wire streaming transfer + side wire streaming transfer (SST). Moreover, the process parameter ranges corresponding to the four modes were summarized. Due to the stable arc and the uniform metal transfer process, SPT and SST can form desirable weld seam. The gravity and z-axis components of electromagnetic force are the main forces that promote metal transfer. The $\mathrm{x}$-axis and $\mathrm{y}$-axis components of the electromagnetic force deviate the metal transfer path from the arc coverage. Due to the change of arc conductive channel, the energy transferred from TW-GIA to the base metal is less than that of GMAW, showing the advantages of small welding deformation, narrow heat affected zone and grain refinement.

Keywords Triple-wire gas indirect arc welding $\bullet$ metal transfer $\bullet$ energy conversion $\bullet$ arc conductive channel 


\section{Introduction}

With the increase in market demand, methods to improve welding efficiency have become the focus of modern industrial research. Increasing deposition rate to reduce welding times is the most direct method [1-3]. For example, Tandem welding [4,5] utilizes two wires to melt synchronously, which significantly improves welding efficiency. T.I.M.E welding [6,7] increases the allowable current by changing the gas composition, and it has been proven that the deposition rate can reach triple times that of the traditional GMAW [8]. However, the prerequisite for these technologies to increase deposition rate is to increase the welding current. The heat input of base metal increases accordingly, which is prone to defects such as undercut [9] and welding deformation [10]. Bypass coupled arc welding [11-13] adopts a bypass system, which allows part of the welding current to pass through the auxiliary wire and the rest to pass through the base metal. This achieves the effect of increasing deposition rate and reducing heat input. Twin-wire indirect arc welding (TWIAW) $[14,15]$ uses the method of separating the base metal from electrode. The positive and negative electrodes of the power are connected by two independent wires, so that the current does not flow through the base metal, and an arc is generated between the wires. The heat-carrying mechanism of base metals is changed to realize the decoupling effect of heat input and deposition rate. However, due to the asymmetrical spatial distribution of the magnetic field, when these technologies use the large current, the arc will diverge severely. The adjustment of process parameters needs to be very precise, which increases the difficulty of the welding process [16].

Recently, a new high-efficiency welding technology called triple-wire gas indirect arc welding(TW-GIA) has been proposed [17]. An indirect arc is generated between the wires, and they are coupled into one body in space. The triple-wire feeding method is adopted to increase the deposition rate, and the base material is separated from the electrode to reduce the heat input. Liu et al. [18] used TW-GIA to weld the thick steel plates of $2 \mathrm{~mm}$ at high speed. The welding speed can reach $2.8 \mathrm{~m} / \mathrm{min}$, which is much higher than traditional GMAW high-speed welding $(<1 \mathrm{~m} / \mathrm{min})$ and bypass coupled arc 
welding $(<1.9 \mathrm{~m} / \mathrm{min})$. In addition, Compared with TWIAW, the action of the spatially symmetrical magnetic field enhances the TW-GIA arc stiffness. Liu et al. [19] explored the process parameter range of TW-GIA, and results showed allowable current can reach more than 480 A (twice of TWIAW). Due to the expansion of the process parameter range, TW-GIA can solve the sidewall non-fusion defect that appears in the thick plate welding process of TWIAW [20].

The high deposition rate is closely related to the metal transfer mode. In traditional GMAW, metal transfer modes can be divided into short-circuit transfer and free transfer. According to droplet shape, the latter can be divided into globular transfer, projected transfer, streaming transition, etc [21-23]. Wu et al. [24] demonstrated different metal transfer modes in DP-GMAW can influence weld appearance and penetration. Ikram et al. [25] explored the force and temperature characteristics of VP-GMAW droplets through numerical simulations, and proved that finger penetration is caused by the falling droplets transferring momentum to the bottom of the molten pool. Madyira et al. [26] showed that different GMAW metal transfer modes under various welding positions will affect the weld mechanical properties. Ogino et al. [27] proved the GMAW metal transfer modes can be altered by adjusting composition of shielding gas.

However, the TW-GIA current forms a loop between the triple wires. The alter of the arc conductive channel changes the space magnetic field. The force mechanism of the droplet has also changed accordingly. In addition, when the triple wires are melted at the same time, their respective metal transfer modes are not the same, which increases the complexity of metal transfer. Moreover, because the base metal is separated from the electrode, the heating mechanism and weld forming characteristics of the base metal are also different from the traditional GMAW. At the present stage, the unclear metal transfer modes and unknown weld forming characteristics limit the development of the TW-GIA process.

This paper aims to clarify the metal transfer mode and weld forming characteristics of TW-GIA. High-speed camera systems and electrical signal acquisition sensors were used to explore the TW-GIA metal transfer mode. The static force model and the arc conductive channel model were used to discuss the droplet force and energy conversion 
characteristics respectively.

\section{Experimental equipment and principles}

The TW-GIA system is mainly composed of two powers and triple independent wire feeding equipments. The wires are arranged in mirror symmetry (Fig. 1b). The middle position is called main wire, and the other two are called side wires. The angle between side wires and main wire is $20^{\circ}$, and the angle between main wire and horizontal is $60^{\circ}$. Extension of the wires is $15 \mathrm{~mm}$. The positive electrodes of two powers are connected to side wires, negative electrodes are concentratedly connected to main wire. Main wire current $I_{M}=I_{L}+I_{R}, I_{L}$ and $I_{R}$ are left and right side wire current respectively. The welding current mentioned in this paper refers to the main wire current. The shielding gas composition is $80 \% \mathrm{Ar}+20 \% \mathrm{CO}_{2}$, and gas flow is $20 \mathrm{~L} / \mathrm{min}$. Q345 steel plate with a size of $500 \mathrm{~mm} \times 160 \mathrm{~mm} \times 3 \mathrm{~mm}$ was adopted as base metal. The welding gap is $2.5 \mathrm{~mm}$, and ceramic gasket on the back was used to constrain the weld pool. The wire is ER50-6, the main wire diameter is $1.6 \mathrm{~mm}$, and the side wire diameter is $1.2 \mathrm{~mm}$. Table 1 shows the chemical composition of wire and base metal.

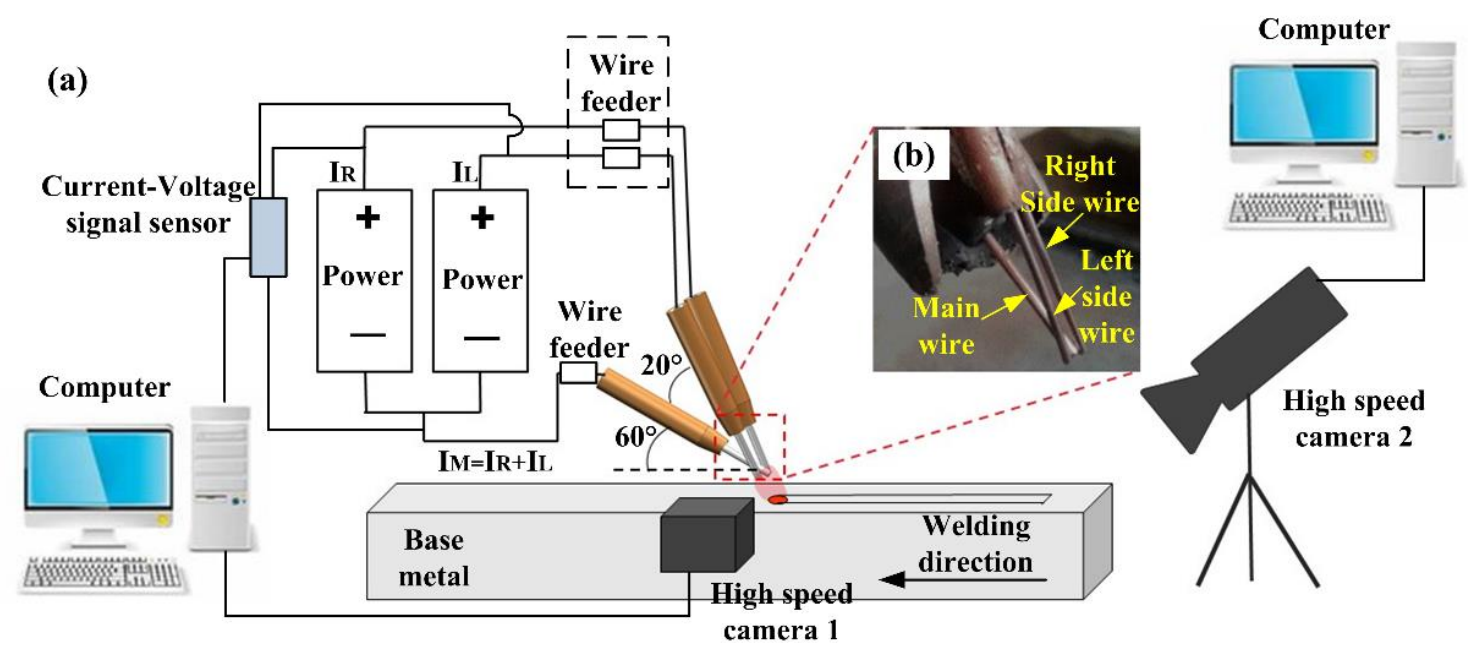

Fig. 1 Schematic diagram of the equipment used

The MS50K high-speed camera produced by Canadian Mega-speed Company was placed to record the behavior of droplet, with a maximum resolution of $1280 \times 1024$ and a spectral range of $400 \mathrm{~nm}-1000 \mathrm{~nm}$. The acquisition frequency is $2000 \mathrm{~Hz}$, and exposure time is $500 \mu \mathrm{s}$. In order to eliminate the interference generated by the arc, a xenon lamp auxiliary light source is equipped during the shooting. The camera lens adds a narrow- 
band filter with a center wavelength of $659.5 \mathrm{~nm}$ to reduce the influence of light of other wavelengths on the captured image. The half-wave bandwidth is $9.4 \mathrm{~nm}$ and the peak transmittance is $58.2 \%$. Hannover welding signal analyzer (AH-19) was used to record the arc voltage and the welding current curve during the welding process. The GMAW welding machine used in the experiment is OTC DP500, equipped with AX-V16 six-axis welding system, which can realize the automatic matching effect of welding current and wire feeding speed.

Table 1. Chemical composition (wt.\%) of wire and base metal

\begin{tabular}{cccccccc}
\hline Materials & $\mathrm{C}$ & $\mathrm{Mn}$ & $\mathrm{Si}$ & $\mathrm{S}$ & $\mathrm{P}$ & $\mathrm{Cu}$ & $\mathrm{Fe}$ \\
\hline $\begin{array}{c}\text { Base metal } \\
\text { Q345 }\end{array}$ & 0.17 & 1.5 & 0.5 & 0.035 & 0.035 & - & Bal. \\
$\begin{array}{c}\text { Welding wire } \\
\text { ER50-6 }\end{array}$ & 0.10 & 1.54 & 0.90 & 0.020 & 0.018 & 0.10 & Bal. \\
\hline
\end{tabular}

\section{Result and discussion}

\subsection{Metal transfer characteristics}

Fig. 2 shows the typical characteristics of the metal transfer path. From the perspective vertical to the welding direction (the two side wires overlap in this perspective) (Fig. 2a), it can be seen that the metal transfer paths of the main wire and the side wire are independent. The transfer path deviates from the extending direction of wires. The parallel direction (Fig. 2b) shows that the side wire droplets repel each other and the transfer path is out of the arc coverage.
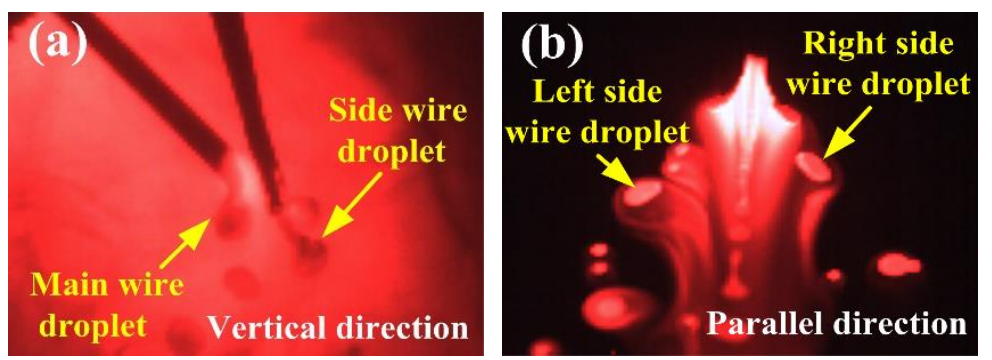

Fig. 2 Typical characteristics of the metal transfer path: (a) the perspective vertical to the welding direction, (b) the perspective parallel to the welding direction

In terms of the metal transfer appearance, since the metal transfer mode of the two side wires is the same, this paper used the electrical signal of the left wire as a representative for display. The modes are mainly divided into the following four types:

(I) Short-circuit transition (SCT): The mode mainly exists in the range of welding 
current less than $200 \mathrm{~A}$ and the arc voltage less than 30 V. Fig. 3 shows the transfer process. The arc conductive channel generated between main wire and side wires, droplets gradually grow up at the wires terminal (Fig. 3a) until the droplets contact each other (Fig. 3b). In TW-GIA, the main wire and side wires are respectively connected to electrodes of two powers. The contact between the droplets makes the welding current loop short-circuit. The sharp drop in arc voltage is accompanied by a sudden increase in welding current. The resistance at the contact point rises thermally, and the liquid temperature quickly reaches the boiling point, causing an explosion (Fig. 3c). The droplet falls under the action of the explosive force (Fig. 3d, Fig. 3e). After the droplet falls off, the arc continues to discharge between the wires. During the whole process, the electrical signal was seriously disturbed.

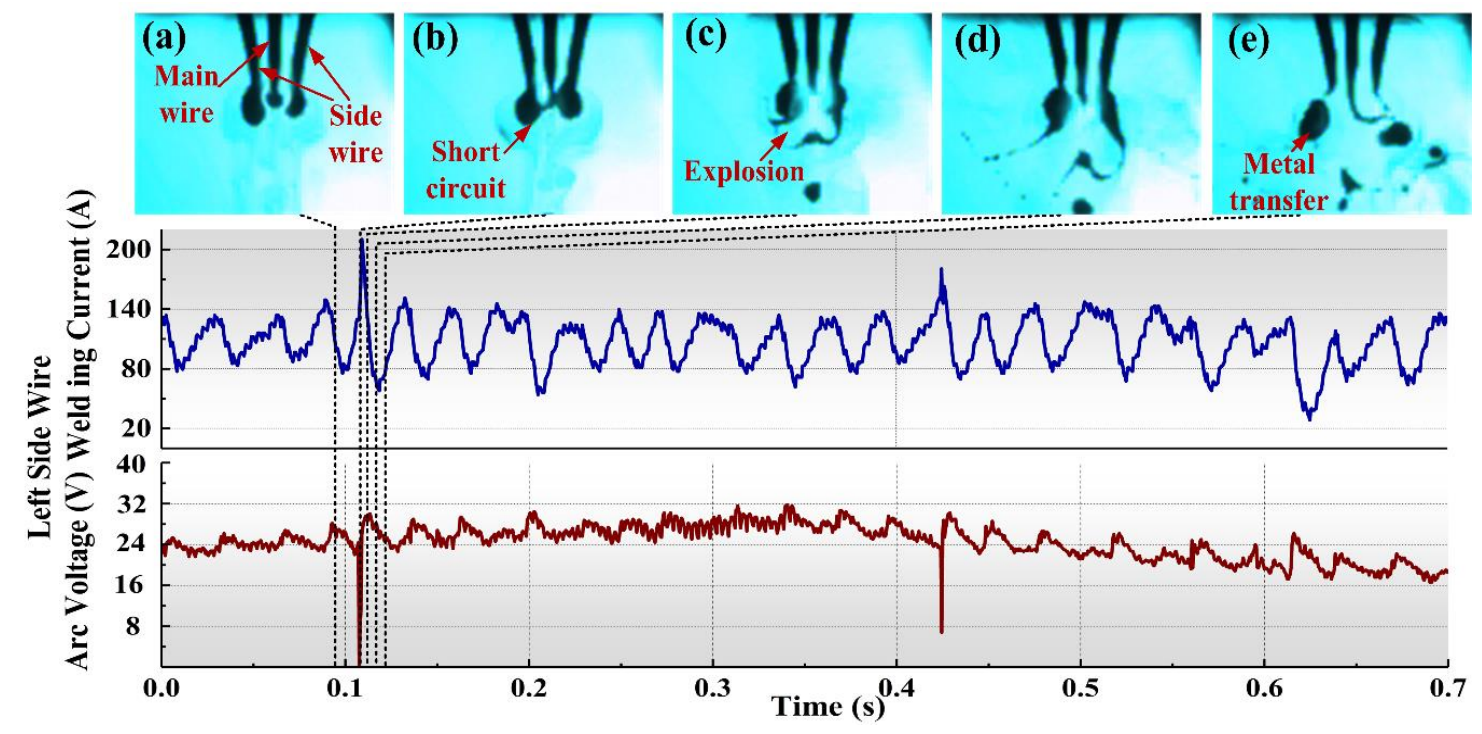

Fig. 3 SCT droplets behavior and electrical signals

(II) Main wire projected transfer + side wire globular transfer (PGT): The mode mainly exists in the range of welding current $200 \mathrm{~A}-270 \mathrm{~A}$ and arc voltage $25 \mathrm{~V}-35 \mathrm{~V}$. Fig. 4 shows the transfer process. At the beginning of welding, an arc is generated between the main wire and the side wires, and the droplets grow up at the end of the wires. As the volume of the droplet increases, the distance between the anode and the cathode is gradually compressed, and the arc voltage decreases accordingly. (Fig. 4a). As the welding progress, the main wire droplet will drop as small spherical liquids (Fig. 4b, Fig. 4c). At the same time, the side wire droplets still remain at the end of the wires 
in a large-volume spherical shape, and the conductive channel is still in a compressed state. Therefore, the arc voltage is still at a relatively low level. The side wire droplets continue to grow with the melting of the wires, until they drop down as a thick sphere with a diameter larger than the wire diameter (Fig. 4d, Fig. 4e). At this time, the arc voltage increases as the increase of the distance between the anode and cathode.

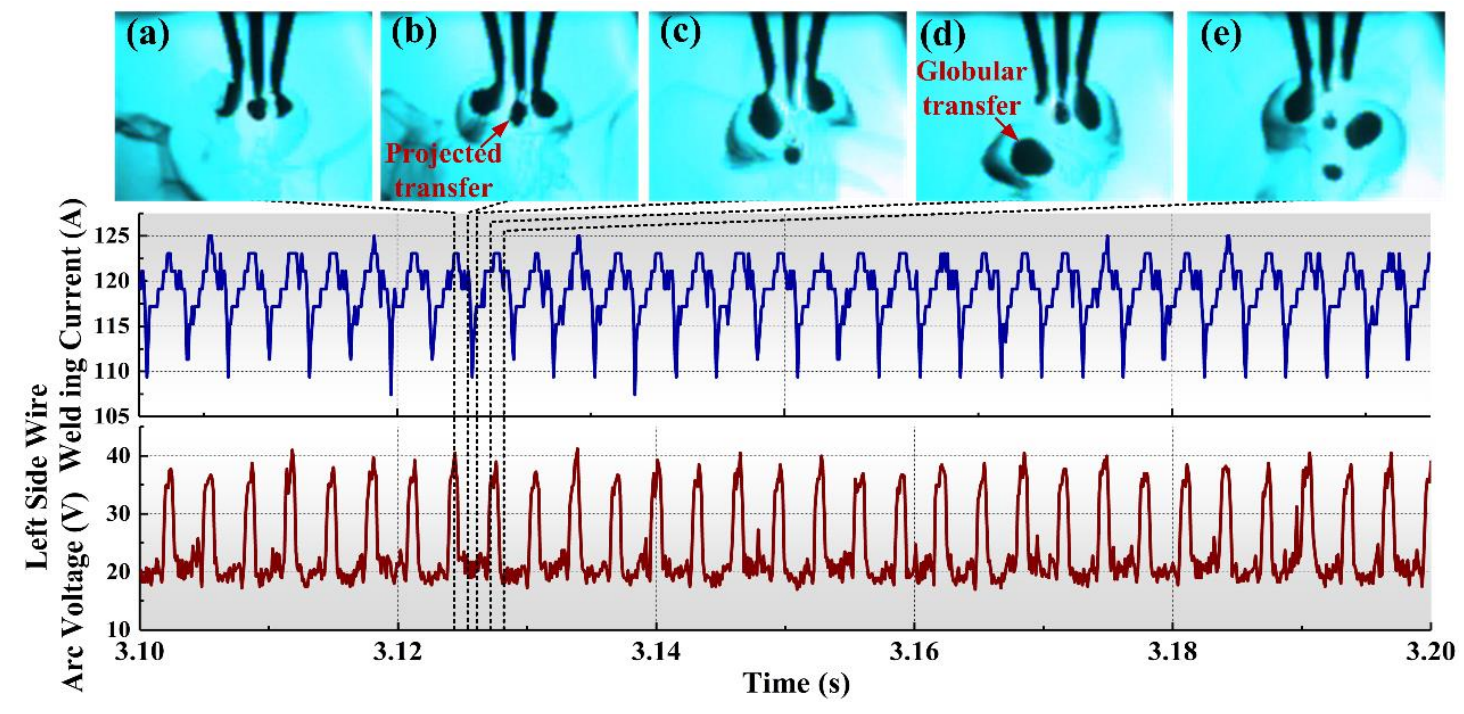

Fig. 4 PGT droplets behavior and electrical signals

(III) Main wire streaming transfer + side wire projected transfer (SPT). The mode mainly exists in the range of welding current 270 A-320 A and arc voltage $25 \mathrm{~V}-35 \mathrm{~V}$. Fig. 5 shows the transfer process. The main wire melts into a continuous column of liquid, and the diameter of the liquid stream is smaller than the wire diameter. The independent fine liquid ball transfer from side wires, and drag-like residual liquid retained at wire terminal. In this mode, the droplets stay on the wires for a short time. It will not alter arc conductive channel and has little influence on the distance between anode and cathode. Therefore, the welding current and arc voltage fluctuate smoothly. 


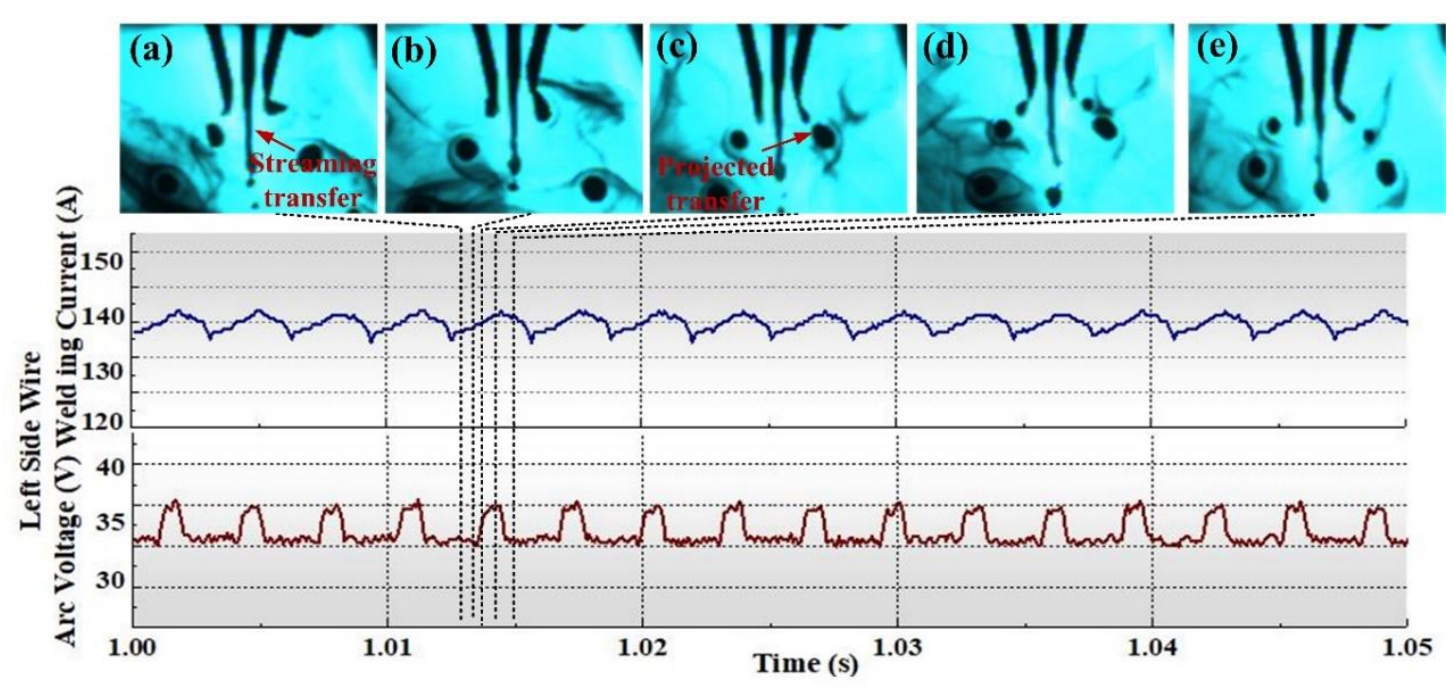

Fig. 5 SPT droplets behavior and electrical signals

(IV) Main wire streaming transfer + side wire streaming transfer (SST): The mode mainly exists in range of welding current exceeds $320 \mathrm{~A}$ and arc voltage exceeds $30 \mathrm{~V}$. Fig. 6 shows the transfer process. The main wire and the side wires are melted into a continuous liquid stream. The diameters of these liquid streams are smaller than their respective wire diameters. And the main wire stream diameter is smaller than that of side wires. Different from the drag-like residual liquid in SPT, the stream length in SST is more than $1 \mathrm{~mm}$.

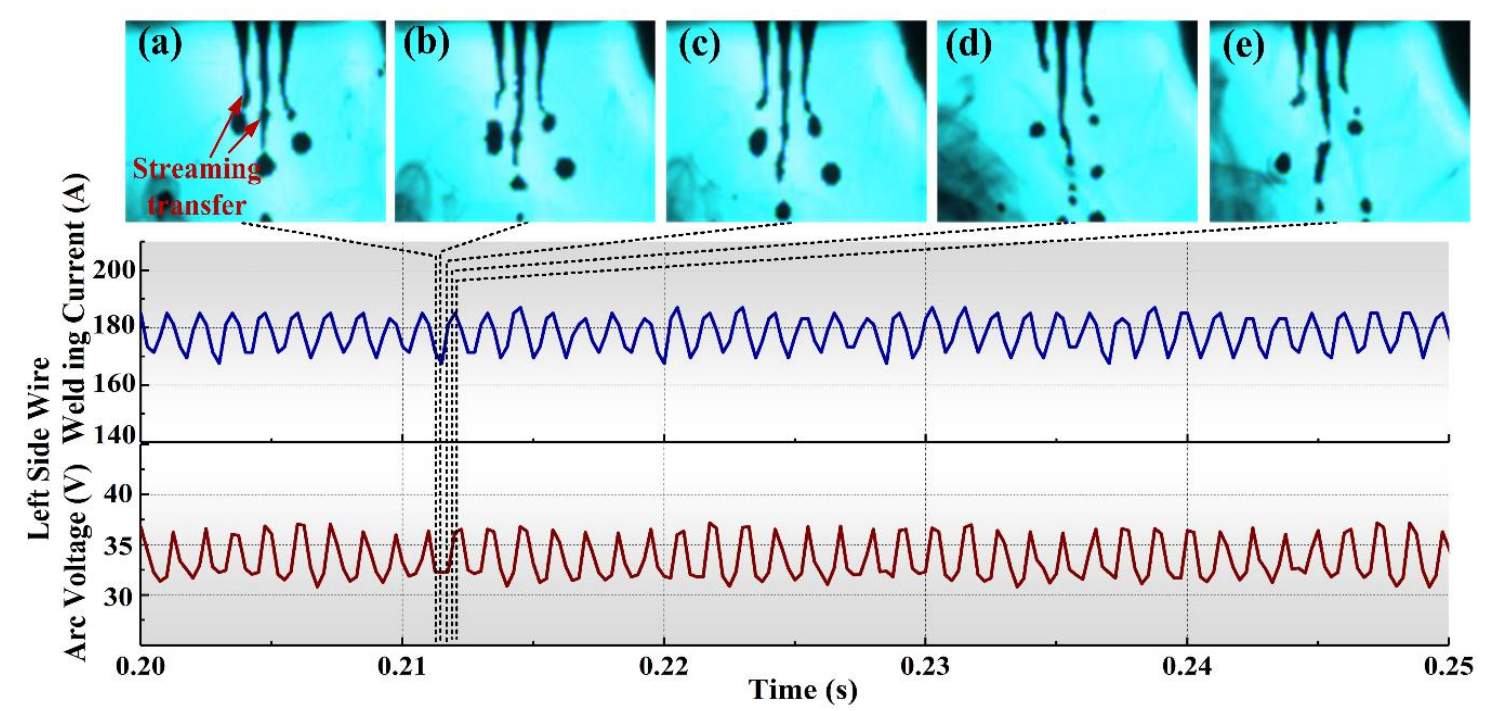

Fig. 6 SST droplets behavior and electrical signals

Besides the above four typical metal transfer modes, other metal transfer modes have also been discovered, such as main wire globular transfer + side wire globular transfer, the main wire projected transfer+ side wire projected transfer, etc. However, these modes only exist in a narrow range at the beginning or ending of the four typical 
metal transfer parameters, which can be regarded as the transition zone of typical modes. Fig. 7 shows the parameter range corresponding to the metal transfer modes, in which the overlapping part of adjacent circles is transition zone.

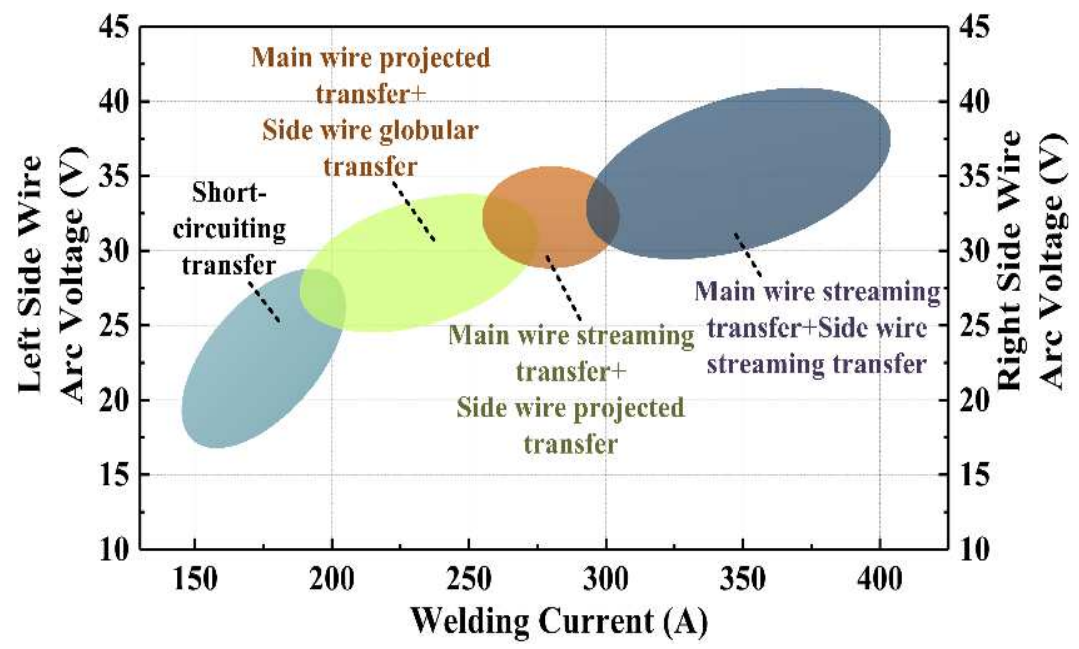

Fig. 7 Parameter range corresponding to TW-GIA metal transfer mode

\subsection{Droplet force analysis}

The metal transfer behavior is closely related to the forces acting on the droplet. For convenience, the droplet is regarded as a standard sphere for static force analysis. Fig. 8 shows the force analysis of the TW-GIA droplets. The types of forces on the main wire and side wires are almost same, mainly including gravity $\boldsymbol{F g}$, surface tension $\boldsymbol{F b}$, spot force $\boldsymbol{F a}$, plasma flow force $\boldsymbol{F p}$ and electromagnetic force (Including the electromagnetic force $\boldsymbol{F s}$ generated by its own wire magnetic field and the electromagnetic force $\boldsymbol{F m}$ generated by other wires magnetic fields).
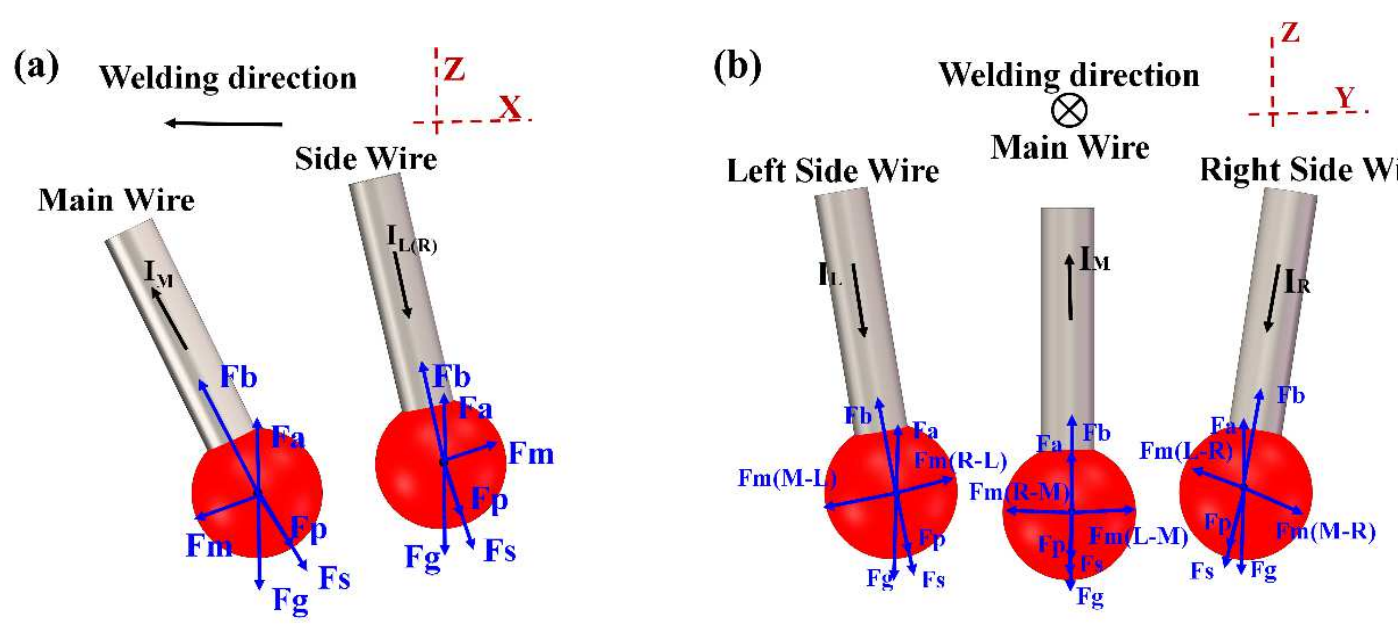

Fig. 8 TW-GIA droplet force model: (a) vertical to the welding direction, (b) parallel to the 
welding direction

The direction of droplet gravity $\boldsymbol{F g}$ is vertically downward. Surface tension $\boldsymbol{F b}$ acts perpendicularly on the circumferential line between the wire terminal and the droplet phase, and has the effect of hindering metal transfer. The spot force $\boldsymbol{F a}$ mainly includes the bombardment force of plasma and the evaporation reaction force of the electrode. When the spot area is smaller than the cross section of droplets, the arc cannot cover the entire droplet. Polar spots mainly exist on the lower surface of droplet. The spot force impinges the droplet in reverse and hinders the metal transfer. When the spot area is larger than the cross section of droplets, the polar spot exists at the wire, the arc covers the entire droplet, and the spot force promotes the metal transfer. It can be seen in Fig. 2 that droplets are not completely wrapped by arc, so the spot force hinders the metal transfer. As a kind of fluid, the arc plasma flow force $\boldsymbol{F} \boldsymbol{p}$ promotes metal transfer.

Electromagnetic force can be calculated by equations 1[28]:

$$
\begin{aligned}
& F_{s}=\frac{\mu I_{s}^{2}}{4 \pi}\left[\ln \frac{r \sin \theta}{r_{w}}-\frac{1}{4}-\frac{1}{1-\cos \theta}+\frac{2}{(1-\cos \theta)^{2}} \ln \frac{2}{1+\cos \theta}\right] \\
& F_{m}=\frac{\mu}{4 \pi} \cdot \frac{I_{s} I_{m}}{d}
\end{aligned}
$$

Where $\mu$ is magnetic permeability in free space, $I_{s}$ is own wire current, $\theta$ is the arc root angle, and $I_{m}$ is current in other wire applying the magnetic field. In the previous report [29], the electromagnetic force of the droplet is much greater than gravity and plasma flow force. From the perspective vertical to welding direction (Fig. 9a), for main wire droplets, the electromagnetic force $\boldsymbol{F s}$ basically coincides with the direction of the current. The electromagnetic force $\boldsymbol{F m}$ includes $\boldsymbol{F m}(\boldsymbol{L}-\boldsymbol{M})$ applied by the left side wire and $\boldsymbol{F m}(\boldsymbol{R}-\boldsymbol{M})$ applied by the right side wire. The direction of the resultant force is perpendicular to the direction of the current at the wire end, and the x-axis component of the electromagnetic force causes the main wire droplet to escape from the arc envelope. Similarly, for the side wire droplets, the $\mathrm{x}$-axis component of the electromagnetic force separates the side wire droplets from the arc coverage, and the $y$ axis component of the electromagnetic force makes the two side wire droplets repel each other, as shown in Fig. 2. 
When welding current is less than $200 \mathrm{~A}$, the z-axis component of electromagnetic force is weak, and the droplet cannot be separated due to the obstacles of surface tension and spot force. The droplets continue to grow at the wire terminals until the wires touch each other and cause a short circuit. When the welding current range is $200 \mathrm{~A}-270 \mathrm{~A}$, the $\mathrm{z}$-axis component of electromagnetic force increases accordingly. In particular, the value of the main wire current is twice that of the single side wire current. The electromagnetic force on the main wire is greater than that on the side wire. The main wire droplet can transfer when the volume is smaller. Side wire droplets gradually grow up at the wire terminal, forming a PGT mode. When the welding current more than $270 \mathrm{~A}$, the strong z-axis component of the electromagnetic force increases the transfer frequency. The droplets are connected together under the action of surface tension, and the boundary of each droplet cannot be distinguished, gradually form the SPT and the SST. Due to the main wire current is greater than that of side wire, the diameter of the main wire liquid stream is smaller than that of the side wire liquid stream.

\subsection{Weld characteristics}

Desirable weld requires stable arc and uniform metal transfer. The arc stability can be evaluated by the electrical signal variation coefficient, which can be calculated by equations 2 :

$$
v=\sqrt{\left[\sum_{i=1}^{n}\left(x_{i}-\bar{x}\right)^{2} \bar{x} /(\mathrm{n}-1)\right]} \times 100 \%
$$

Where $v$ is the electrical signal variation coefficient, $\bar{x}$ is the average, and $\boldsymbol{x}_{\boldsymbol{i}}$ is the value of the i-th sample. Fig. 9 shows the variation coefficient of TW-GIA process parameters and the corresponding typical weld appearance. With the increase of welding parameters, variation coefficient shows an overall downward trend. However, in the transition zone of the typical modes, arc fluctuations lead to a local increase in the variation coefficient. Table 2 shows the average current/voltage variation coefficient of the different metal transfer modes. The variation coefficient of SCT and PGT significantly higher than that of SPT and SST, which means the arc instability of SCT 
and PGT is greater. And in SCT, the explosive force causes the droplets to diverge and cannot be accurately transferred to the predetermined weld track. In PGT, intermittent large droplets will impact the molten pool and form hump defects [30]. Therefore, due to the unstable arc and the turbulent metal transfer process, SCT and PGT cannot form the desirable weld seam. While the arc in SPT and SST is stable, and droplets are tiny and uniform, which is prone to satisfactory weld seam.

In order to explore the practicality of TW-GIA, the butt weld of TW-GIA and GMAW under the same parameters (Welding current 280 A, welding speed 900 $\mathrm{mm} / \mathrm{min}$ ) were compared. Fig. 10 shows the weld appearance and microstructure of the two processes. The TW-GIA weld (Fig. 10a) has no obvious defects, and the GMAW weldment has severely bending deformation (Fig. 10b). The average width of TW-GIA heat-affected zone (HAZ) (Fig. 10c) is about $1.9 \mathrm{~mm}$, and GMAW is about $3.7 \mathrm{~mm}$ (Fig 10f). The HAZ (Fig. 10d) and fusion zone (FZ)(Fig. 10e) of TW-GIA are mainly composed of fine pre-eutectoid ferrite, carbide and pearlite. The GMAW grain size is significantly larger than the grains at the same position in TW-GIA, and coarse widmanite structure appears at the GMAW grain boundaries (Fig. 10g). The side plate ferrite structure growing along the GMAW grain boundary in FZ (Fig. 10h). The above results showed TW-GIA has the advantage of low heat input than GMAW.

Table 2 The average variation coefficient (\%) of the different metal transfer modes

\begin{tabular}{ccccc}
\hline & SCT & PGT & SPT & SST \\
\hline $\begin{array}{c}\text { Coefficient of current } \\
\text { variation }\end{array}$ & 39.1 & 23.4 & 8.9 & 6.3 \\
$\begin{array}{c}\text { Coefficient of voltage } \\
\text { variation }\end{array}$ & 83.5 & 65.0 & 39.5 & 32.8 \\
\hline
\end{tabular}




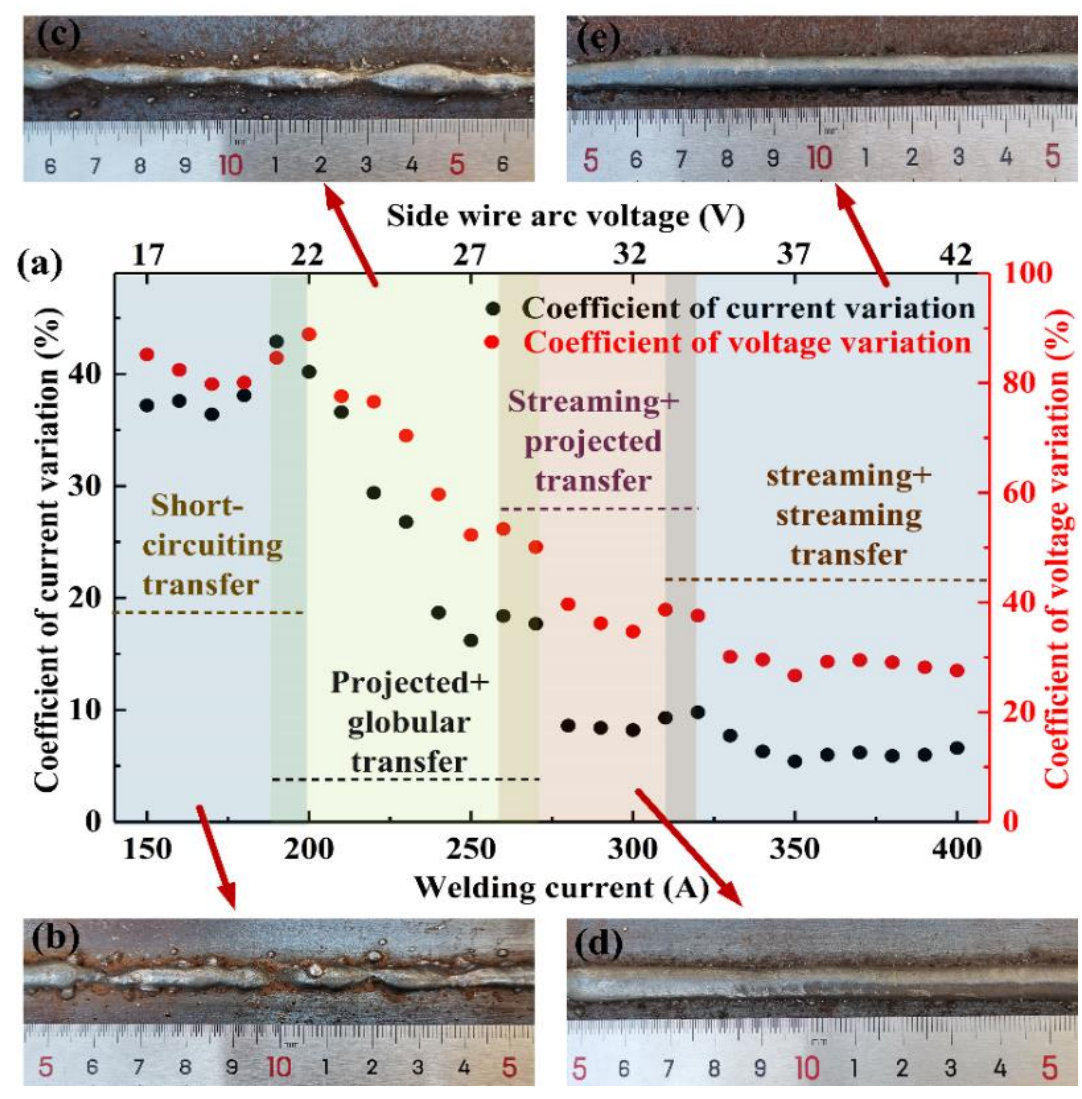

Fig. 9 Variation coefficient and typical weld appearance of different TW-GIA process parameters:

(a) variation coefficient of current and voltage, (b) SCT mode, (c) PGT mode, (d) SPT mode, (e)

SST mode.
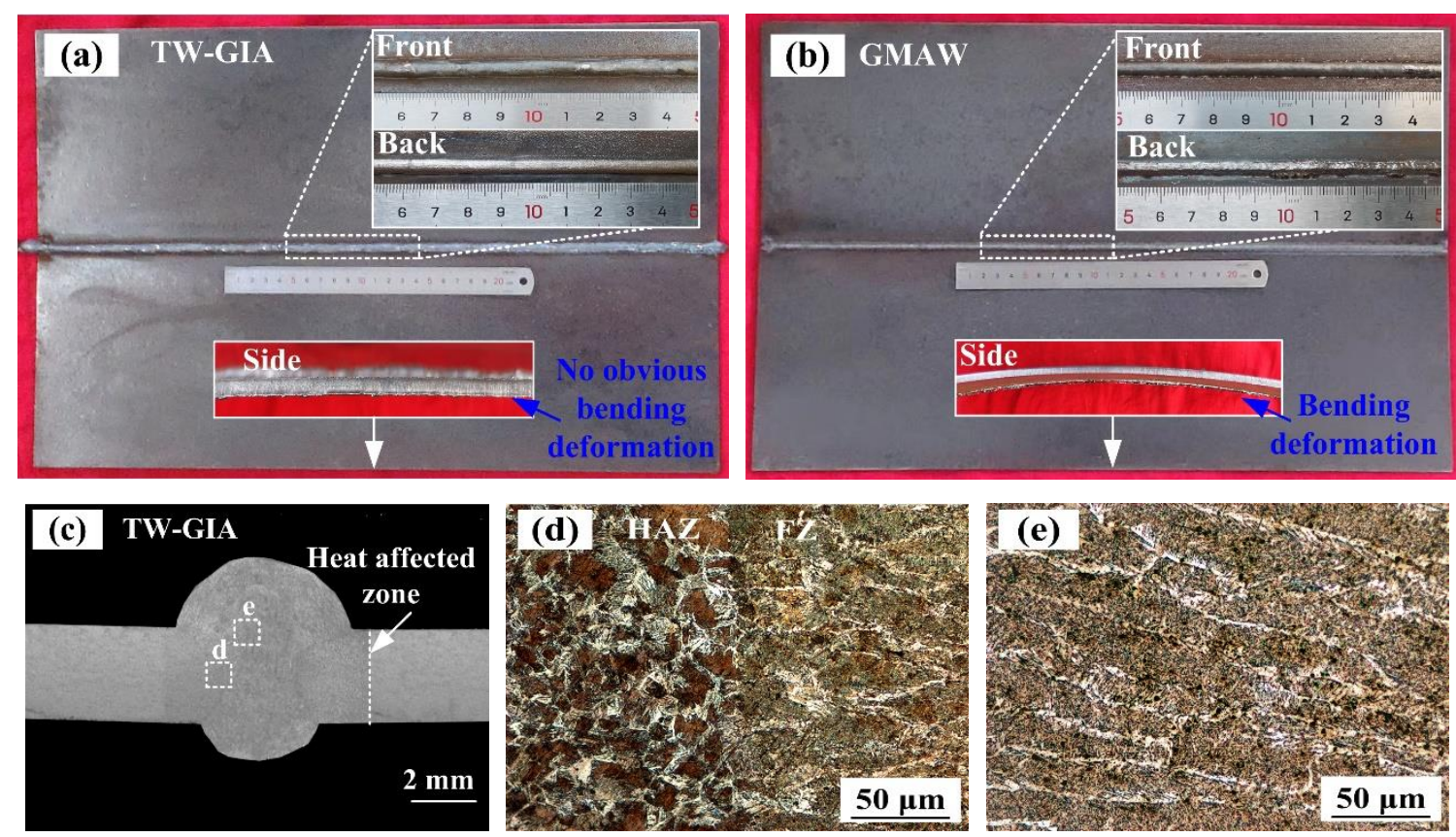

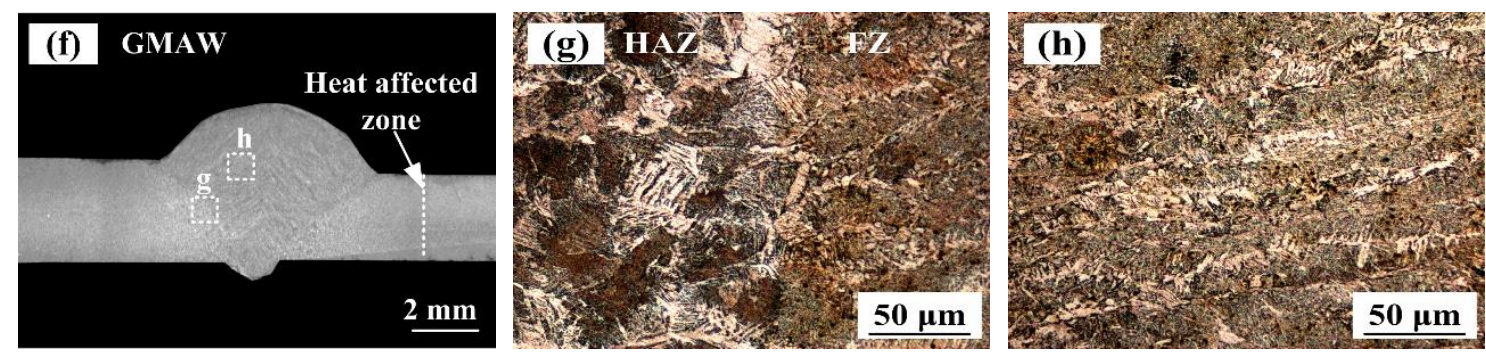

Fig. 10 TW-GIA and GMAW weld appearance and microstructure comparison: (a) TW-GIA weld appearance, (b) GMAW weld appearance, (c) TW-GIA weld cross section, (d) GMAW weld cross section, (e) TW-GIA HAZ microstructure, (f) TW-GIA FZ microstructure, (g) GMAW HAZ microstructure, (h) GMAW FZ microstructure.

Fig. 11 shows the tensile properties of TW-GIA weld. The fracture location away from FZ. The tensile strength is $459 \mathrm{MPa}$, which is consistent with the base metal. The elongation is $24.7 \%$, which shows the TW-GIA process is suitable for butt welds of 3 $\mathrm{mm}$ thick low carbon steel with excellent mechanical properties.

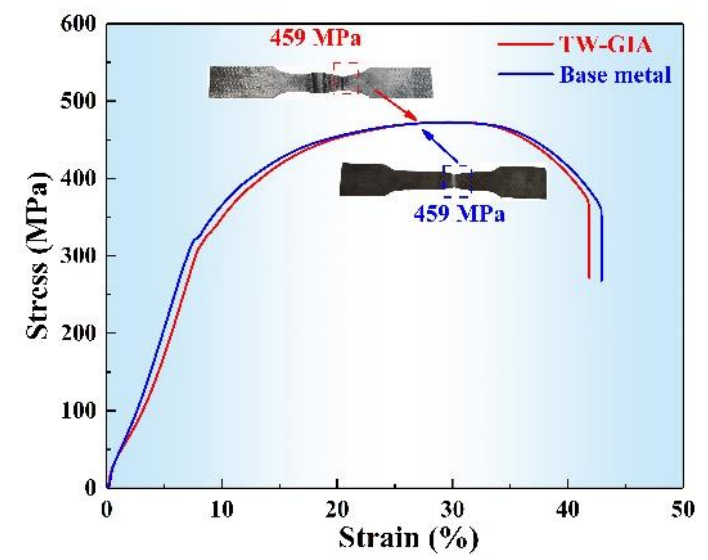

Fig. 11 Tensile properties of TW-GIA weld

\subsection{Energy conversion analysis}

The difference in heat input between the two processes is mainly caused by the difference in energy conversion mechanism. The replacement of the TW-GIA arc conduction channel changes the energy conversion characteristics of base metals, as shown in Fig. 12. 

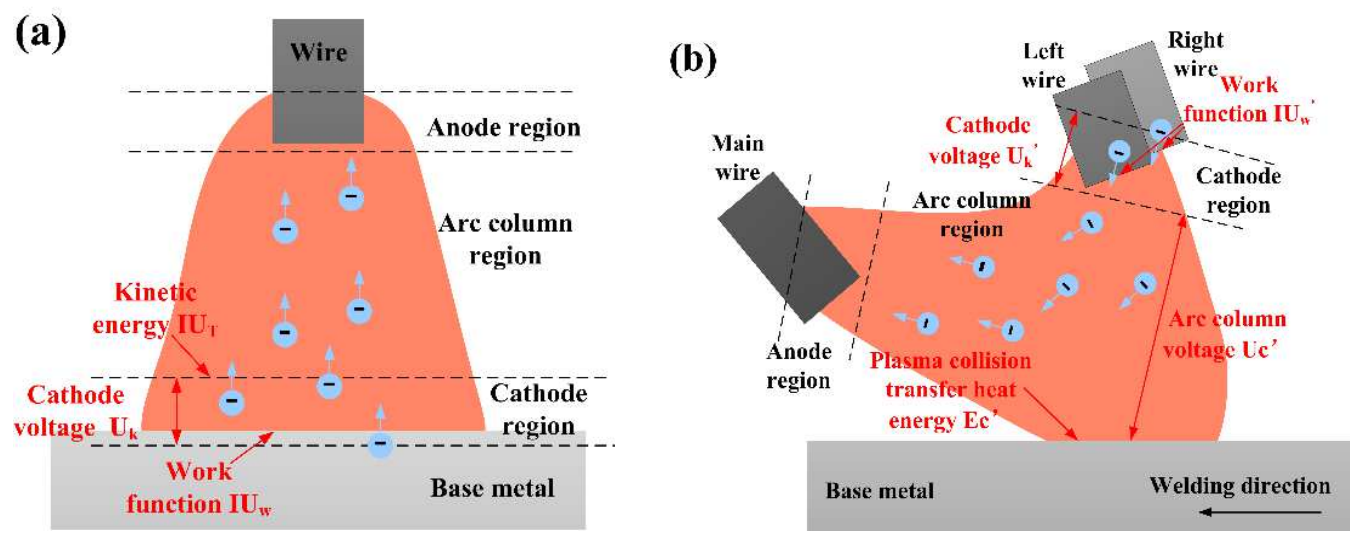

Fig. 12 Arc conductive channel comparison: (a) GMAW, (b) TW-GIA

In GMAW (Fig. 12a), the base metal is connected to the cathode, and the received heat energy comes from the arc plasma. The positive ions provided by the arc column region to cathode region only account for $0.1 \%$, and it can be negligible to the heat production. Therefore, the arc plasma that affects the energy state of base metal is entirely generated in cathode region. Under the premise of ignoring the minimal heat loss due to heat convection with air and heat radiation, the energy conversion of GMAW base metal mainly comes from the following four aspects:

1) The energy $\boldsymbol{I} \boldsymbol{U}_{\boldsymbol{k}}$ obtained by electrons under the action of cathode voltage, $\boldsymbol{U}_{\boldsymbol{k}}$ is value of cathode voltage.

2) The work function $\boldsymbol{I} \boldsymbol{U}_{\boldsymbol{W}}$ consumed when electrons overflow the cathode surface, $\boldsymbol{U}_{W}$ is escape voltage.

3 ) The kinetic energy $\boldsymbol{I} \boldsymbol{U}_{\boldsymbol{T}}$ taken away by electrons from the cathode region into arc column region, $\boldsymbol{U}_{\boldsymbol{T}}$ is arc column temperature equivalent voltage.

4) The heat energy $\boldsymbol{E}_{\boldsymbol{d}}$ carried by the droplets.

The heat energy $\boldsymbol{P}_{\boldsymbol{K}}$ of the GMAW base metal conforms to equations 3:

$$
\mathrm{P}_{\mathrm{K}}=\mathrm{I}\left(\mathrm{U}_{\mathrm{k}}-\mathrm{U}_{\mathrm{W}}-\mathrm{U}_{\mathrm{T}}\right)+\mathrm{E}_{\mathrm{d}}
$$

When electrons enter the arc column region, they will collide with other neutral ions that move irregularly. Most kinetic energy is converted into heat energy. The more frequent the collision between electrons and neutral ions, the more heat is converted, and the kinetic energy $\boldsymbol{I} \boldsymbol{U}_{\boldsymbol{T}}$ smaller. When the arc temperature is $6000 \mathrm{~K}, \boldsymbol{U}_{\boldsymbol{T}}<1 \mathrm{~V}[30]$. Therefore, the formula 3 can be simplified to equations 4 :

$$
\mathrm{P}_{\mathrm{K}}=\mathrm{I}\left(\mathrm{U}_{\mathrm{k}}-\mathrm{U}_{\mathrm{W}}\right)+\mathrm{E}_{\mathrm{d}}
$$


In TW-GIA (Fig. 12b), the base metal is not connected to the electrodes and therefore does not withstand the heat generated in the cathode region. The Electrons collision and droplet heat are the main sources of heat input. Due to the length of arc cathode and anode region is small, generally $10^{-3} \mathrm{~cm}-10^{-2} \mathrm{~cm}$, the electrons that collide with base metal are mainly in arc column region. So, the heat energy of TW-GIA base metal mainly includes:

1) The energy $\boldsymbol{j} \boldsymbol{A}\left(\boldsymbol{U}_{\boldsymbol{k}}{ }^{\prime}-\boldsymbol{U}_{\boldsymbol{W}^{\prime}}\right)$ obtained when the electrons leave cathode region, $\boldsymbol{j}$ is the collision current density, $\boldsymbol{A}$ is collision area, $\boldsymbol{U}_{\boldsymbol{k}}{ }^{\prime}$ is value of cathode voltage, and $\boldsymbol{U}_{\boldsymbol{W}^{\prime}}$ is escape voltage.

2) The energy $\boldsymbol{j} \boldsymbol{A} \boldsymbol{U}_{\boldsymbol{C}^{\prime}}$ gained by electrons accelerating in arc column region, $\boldsymbol{U}_{\boldsymbol{C}}{ }^{\prime}$ is arc column voltage experienced by electrons.

3 ) Irregularly moving plasma collision transfer heat energy $\boldsymbol{E}_{\boldsymbol{C}^{\prime}}$ in arc column region.

4) The heat energy $\boldsymbol{E}_{\boldsymbol{d}}{ }^{\prime}$ carried by droplets.

So the heat energy $\boldsymbol{P}_{T}$ of TW-GIA base metal conforms to equations 5:

$$
\mathrm{P}_{\mathrm{T}}=\mathrm{j} \mathrm{A}\left(\mathrm{U}_{\mathrm{k}}{ }^{\prime}-\mathrm{U}_{\mathrm{W}^{\prime}}+\mathrm{U}_{\mathrm{C}^{\prime}}\right)+\mathrm{E}_{\mathrm{C}^{\prime}}+\mathrm{E}_{\mathrm{d}^{\prime}} \quad(5)
$$

According to the principle of minimum voltage, the TW-GIA conductive channel is preferentially established at the minimum distance between the main wire and the side wires, and the current density is dense between the triple wires and gradually decreases toward the edge of the arc. The TW-GIA base metal cannot completely block the conductive channel, and can only be contacted in a small area under the arc. Therefore, the number of electrons per unit time of collision of TW-GIA base metal is much smaller than GMAW welding current $(\boldsymbol{j} \boldsymbol{A}<\boldsymbol{I})$. The welding current of TW-GIA is divided equally by two side wires. So, the number of electrons emitted from the cathode region of a single sided wire can be considered as half of the total number of electrons. Under the same welding current, the plasma density in the cathode region of GMAW is greater than that of TW-GIA, which causes the cathode voltage to increase $\left(\boldsymbol{U}_{\boldsymbol{k}}{ }^{\prime}<\boldsymbol{U}_{\boldsymbol{k}}\right)$. The work function of electrons is related to the material properties and surface state of the wires. The materials of two processes are the same. Therefore, it can be considered that there is no significant difference in the work function $\left(\boldsymbol{U}_{\boldsymbol{W}} \approx \boldsymbol{U}_{\boldsymbol{W}}{ }^{\prime}\right)$. TW-GIA 
directional moving electrons do not experience the entire arc column before hitting base metal, but only experience partial column acceleration. The corresponding arc column voltage $\boldsymbol{U}_{\boldsymbol{C}^{\prime}}$ conforms to equations 6 :

$$
\begin{aligned}
\mathrm{U}_{\mathrm{C}}^{\prime} & =\mathrm{I}\left(\mathrm{L}_{\mathrm{C}} / \mathrm{S}_{\mathrm{C}} \gamma_{\mathrm{C}}\right) \\
& =\mathrm{j}\left(\mathrm{L}_{\mathrm{C}} / \gamma_{\mathrm{C}}\right)
\end{aligned}
$$

$\boldsymbol{L}_{\boldsymbol{C}}$ is the length of arc column, $\boldsymbol{S}_{\boldsymbol{C}}$ is cross-sectional area of arc column, and $\boldsymbol{\gamma}_{\boldsymbol{C}}$ is conductivity. It proves the arc column voltage experienced by electrons is related to electron density at the collision. During TW-GIA welding, the base metal contacts arc edge, electron density $\boldsymbol{j}$ is small. The arc edge temperature is low. It can be considered that the arc column voltage $\boldsymbol{U}_{\boldsymbol{C}^{\prime}}$ and the transferred heat $\boldsymbol{E}_{\boldsymbol{C}}{ }^{\prime}$ are both at a relatively low level. In addition, the droplet temperature is limited by the melting point and the boiling point. The energy transferred by the droplets of the two processes is basically the same $\left(\boldsymbol{E d} \approx \boldsymbol{E} \boldsymbol{d}^{\prime}\right)$.

In summary, at the same welding current, $\boldsymbol{P}_{\boldsymbol{T}}<\boldsymbol{P}_{\boldsymbol{A}}$. The energy transferred from TW-GIA to the base metal is less than that of GMAW, showing the advantage of low heat input.

\section{Conclusion}

In this study, the TW-GIA metal transfer mode and the weld forming characteristics were studied. The reasons of TW-GIA low heat input was discussed by establishing an energy conversion model. The following conclusions were drawn:

1) TW-GIA metal transfer modes can be divided into: short-circuit transfer (SCT), main wire projected transfer + side wire globular transfer (PGT), main wire streaming transfer + side wire projected transfer $(\mathrm{SPT})$ and main wire streaming transfer + side wire streaming transfer (SST). Moreover, the process parameter ranges corresponding to the four modes were summarized. Due to the stable arc and the uniform metal transfer, SPT and SST are more suitable to form a desirable weld seam.

2) The gravity and z-axis components of electromagnetic force are the main forces that promote metal transfer. The $\mathrm{x}$-axis and $\mathrm{y}$-axis components of the electromagnetic force deviate the metal transfer path from the arc coverage.

3) The replacement of the TW-GIA arc conduction channel changes the energy 
conversion characteristics of base metals. The cathode voltage, the number of plasma at collision and the arc column voltage experienced by TW-GIA electrons are all at low level. As a result, the energy transferred from TW-GIA to the base metal is less than that of GMAW, showing the advantages of small welding deformation, narrow heat affected zone and grain refinement.

\section{Authors' contribution}

Liming Liu: conceptualization, writing-review and editing, funding acquisition.

Zeli Wang: methodology, writing-original draft, formal analysis.

Tianyi Zhang: assist experiment, validation.

Xianli Ba: conceptualization.

Funding This work was supported by the National Natural Science Foundation of China (No. 52175290).

Data availability All the data have been presented in the manuscript.

\section{Declarations}

Competing interests The authors declare that they have no conflict of interests.

Ethical approval The paper follows the guidelines of the Committee on Publication Ethics(COPE).

Consent to participate The authors declare that they all consent to participate this research.

Consent to publish The authors declare that they all consent to publish the manuscript.

\section{References}

[1] Bashkar R, Balasubramanian V, Mani C, Sonar T (2021) Establishing empirical relationship between welding current and weld metal deposition rate for submerged arc welding process. Multiscale and Multidisciplinary Modeling, Experiments and Design 4:275-291.

[2] Wu D T, Zou Y, Zhao G L, Shi C W (2020) Wear-Resistant Surfacing Layer Preparated by High Efficiency Twin-Wire Indirect Arc Welding. Materials Science Forum 985:229-239.

[3] Wang Y, Yu C , Lu H , Chen J (2020) Research status and future perspectives on ultrasonic arc welding technique. J Manuf Process 58:936-954.

[4] Voiculescu I, Geanta V, Rusu C, Mircea O, Mistodie R L, Scutelnicu E (2016) Research on the metallurgical behaviour of X70 steel subjected to multi wire submerged arc welding.Annals of the University 'Dunarea de Jos' of Galati: Fascicle XI 27:38-46.

[5] Wu K Y, Yin T, Ding N, Zeng M, Liang Z Y (2018) Effect of phase on the behavior of metal transfer in double-wire pulsed GMAW. Int J Adv Manuf Tech 97(9-12):3777-3789.

[6] Fang Y J, Jiang X S, Mo D F, Zhu D G, Luo Z P (2019) A review on dissimilar metals' welding 
methods and mechanisms with interlayer. Int J Adv Manuf Tech 2019;102: 2845-2863.

[7] Lahnsteiner R. T.I.M.E. process - an innovative MAG welding process (1992) Weld Re Int 11(1):17-20.

[8] Layus P, Kah P, Martikainen J, Gezha V V, Bishokov R V (2014) Multi-wire SAW of 640MPa Arctic shipbuilding steel plates. Int J Adv Manuf Tech 75(5-8):771-782.

[9] Chen J, Zhang Y, Wu C S, Padhy G K (2019) Suppression of undercut defects in high-speed GMAW through a compound magnetic field. J Mater Process Tech 274:1-11.

[10] Lu Y, Lu C, Zhang D, Chen Y, Zeng J, Wu P (2019) Numerical computation methods of welding deformation and their application in bogie frame for high-speed trains. J Manuf Process 38:204-213.

[11] Huang J K, Han R H, Shi Y, Fan D, Zhang Y M (2012) Force Analysis of Metal Transfer in Dual Bypass MIG Welding. J Mech Engineering 48(8):44-52.

[12] Zhang Y M, Pan C, Male AT (2000) Improved microstructure and properties of 6061 aluminum alloy weldments using a double-sided arc welding process. Metall Mater Trans A 31(10):253743.

[13] Zhang Y M, Jiang M, Lu W (2004) Double electrodes improve GMAW heat input control. Weld J 83(11):39s-41s.

[14] Lu Y, Chen S J, Shi Y, Li X R, Chen An Q, Wen Y X, Matsuda J (2021) Corrosion resistance and high temperature wear behavior of carbideenhanced austenite-based surfacing layer prepared by twin-wire indirect arc welding. Mater Res Express 8:1-11.

[15] Shi C , Zou Y, Zou Z ,Wu D T (2014) Twin-wire indirect arc welding by modeling and experiment. J Mater Process Tech 214(11):2292-2299.

[16] Wu D T, Hu C, Zhao W, Zhang Y G, Zou Y (2019) Influence of external magnetic field on twin-wire indirect arc surfacing stainless steel layer. Vacuum 169:1-7.

[17] Fang D S, Song G, Liu L M (2016) A Novel Method of Triple-Wire Gas Indirect Arc Welding. Mater Manuf Process 31(3): 352-358.

[18] Liu L M, Yu S B, Song G, Hu C H (2019) Analysis of arc stability and bead forming with highspeed TW-GIA welding. J Manuf Process 46:67-76.

[19] Liu L M, Hu C H, Yu S B, Song G (2019) A triple-wire indirect arc welding method with high melting efficiency of base metal. J Manuf Process 44: 252-260.

[20] Liu L M, Yu S B, Hu C H (2019) Analysis of arc shape and weld forming in triple-wire indirect arc welding. Transactions of the China Welding Institution 40(6): 1-6.

[21] Xu G , Hu J , Tsai H L (2009) Triple-dimensional modeling of arc plasma and metal transfer in gas metal arc welding. Int J Heat Mass Tran 52(7-8):1709-1724.

[22] Ueyama T, Ohnawa T, Tanaka M, Nakata K (2013) Effects of torch configuration and welding current on weld bead formation in high speed tandem pulsed gas metal arc welding of steel sheets. Sci A Tech of Welding \& Joining 10(6):750-759.

[23] Meng X , Qin G , Zhang Y Q, Fu B L, Zou Z D (2014) High speed TIG-MAG hybrid arc 
welding of mild steel plate. J Mater Process Tech 214(11):2417-2424.

[24] Wu K, Cao X, Yin T, Zeng M, Liang Z (2019) Metal transfer process and properties of doublewire double pulsed gas metal arc welding. J Manuf Process 44:367-375.

[25] Ikram A, Chung H (2021) Numerical simulation of arc, metal transfer and its impingement on weld pool in variable polarity gas metal arc welding. J Manuf Process 64:1529-1543.

[26] Daniel M. Madyira, Abdullah Kaymakci, Ntokozo Nkwanyana (2020) The effect of metal transfer modes on mechanical properties of 3CR12 stainless steel. T Cac Soc Mech Eng 44:115.

[27] Ogino Y, Hirata Y, Asai S (2020) Discussion of the Effect of Shielding Gas and Conductivity of Vapor Core on Metal Transfer Phenomena in Gas Metal Arc Welding by Numerical Simulation. Plasma Chen Plasma P 40(5):1109-1126.

[28] Zhu M, Shi Y, Fan D (2015) Analysis and Improvementof M etal Transfer Behaviors in Consumable Double-Electrode GMAW Process. J Manuf Sci E-T ASME 137:1-5.

[29] Zhao Y, Chung H (2017) Numerical simulation of droplet transfer behavior in variable polarity gas metal arc welding. Int J Heat Mass Tran 111:1129-1141.

[30] Wang Z J (2007) Fusion welding method and equipment. Machinery Industry Press, Beijing. (in Chinese) 